\title{
Diferencias transculturales en la declaración de impuestos ante diferentes niveles de presión fiscal: Evidencia experimental con muestras de Argentina y España
}

\author{
Cross cultural differences in tax compliance behavior at different tax \\ rates: Experimental evidence with samples from Argentina and Spain
}

\author{
Lucas Tanos Robein ${ }^{1}$, Bianca Saavedra ${ }^{2}$, Débora Mola ${ }^{3}$ y Cecilia Reyna ${ }^{4}$ \\ Universidad Nacional de Córdoba - Argentina
}

Recibido: $06-02-20$

Aceptado: $12-06-20$

\section{Resumen}

El objetivo fue analizar el impacto de contextos socio-económicos diferentes sobre la declaración de ganancias ante distintos niveles de presión fiscal. Adicionalmente, se examinó el efecto de la fuente del dinero. En el primer estudio, estudiantes de Argentina y España realizaron una tarea de resolución de anagramas para generar ganancias de manera endógena y declararon sus ganancias ante distintos niveles de presión fiscal. En el segundo estudio, estudiantes de Argentina dispusieron de dinero experimental al inicio del estudio y también indicaron su declaración ante distintos niveles de presión fiscal. Se observaron diferencias significativas entre los participantes de Argentina y España en altos índices de presión fiscal. Además, cuando el dinero era ganado los participantes declararon significativamente más en comparación a cuando era brindado por el experimentador. Esto resalta la importancia de considerar aspectos socioculturales al definir las políticas fiscales y el impacto de la fuente del dinero en la declaración.

Palabras clave: Impuestos; transcultural; presión fiscal; esfuerzo.

\footnotetext{
${ }^{1}$ Licenciado en psicología. Cursado finalizado de Maestría en Dirección de Negocios. Trabaja actualmente en relación de dependencia, Universidad Nacional de Córdoba. E-mail: lucastanos@gmail.com

ORCID: https://orcid.org/0000-0002-1595-3002

${ }^{2}$ Licenciada en psicología. Estudiante de Posgrado en la facultad de Psicología, Universidad Nacional de Córdoba; trabajadora independiente. E-mail: saavedrabiancaa@gmail.com

ORCID: https://orcid.org/0000-0003-2627-5945

${ }^{3}$ Doctora en Psicología. Docente en la facultad de Psicología, Universidad Nacional de Córdoba; becaria postdoctoral en el IIPsi - UNC - CONICET. E-mail: debora.mola@unc.edu.ar

ORCID: https://orcid.org/0000-0002-7810-2424

${ }^{4}$ Doctora en Psicóloga. Docente en la facultad de Psicología, Universidad Nacional de Córdoba; investigadora en el IIPsi - UNC - CONICET. Autora corresponsal. E-mail: ceciliareyna@unc.edu.ar ORCID: https://orcid.org/0000-0002-6097-4961
}

(C) Los autores. Este artículo es publicado por la Revista de Investigación en Psicología de la Facultad de Psicología, Universidad Nacional Mayor de San Marcos. Este es un artículo de acceso abierto, distribuido bajo los términos de la licencia Creative Commons Atribucion - No Comercia_Compartir Igual 4.0 Internacional. (http://creativecommons.org/licenses/by-nc-sa/4.0/) que permite el uso no comercial, distribución y reproducción en cualquier medio, siempre que la obra original sea debidamente citada. 


\begin{abstract}
The aim of this study was to analyze the role of different socioeconomic backgrounds in tax compliance behavior at different tax rates. Additionally, we examine the effect of the source of income (whether if it is earned or endowed) on tax behavior. In the first study, students from Argentina and Spain performed an anagram task to earn profits and then they declared their earnings at different tax rates. In the second study, students from Argentina were endowed with an income at the beginning of the experiment and then they declared their earnings at different tax rates. Significant cross-cultural differences were observed at high tax rates. Also, when income was earned, participants declared significant more than when de income was endowed. The results show the importance to consider sociocultural aspects when making public policy and the role of income source in tax behavior.
\end{abstract}

Keywords: Tax; cross cultural; tax rates; effort.

El funcionamiento del Estado depende, en gran parte, de las contribuciones de sus ciudadanos, con las que se busca satisfacer las necesidades colectivas e implementar políticas redistributivas. La evasión fiscal es un problema presente en numerosos países y ha ganado progresiva atención en el campo académico volviéndose prioritario en la agenda de políticas públicas. Diversas investigaciones han demostrado la incidencia de distintas variables sobre el comportamiento de declaración de impuestos, tanto económicas (p.e., la variación de los niveles de presión fiscal, Slemrod \& Yitzhaki, 2002), como psicológicas (p.e., el modo de obtención del ingreso, Sagmeister, Muehlbacher, \& Kirchler, 2009), culturales (p.e. Hyun, 2006; Kogler et al., 2013; Lewis, Carrera, Cullis, \& Jones, 2009) y sociodemográficas (Hofmann, Voracek, Bock, \& Kirchlerb, 2017). En pos de contribuir a ese campo de conocimiento, en esta investigación analizamos el efecto de diferentes niveles de presión tributaria sobre el comportamiento fiscal en ciudadanos argentinos y españoles. También evaluamos el impacto de la forma de obtención de dinero (con vs. sin esfuerzo) sobre la declaración impositiva. A continuación, se definen las variables centrales para esta investigación y las hipótesis derivadas de los antecedentes. En el apartado siguiente, se mencionan los aspectos metodológicos y, luego, se exponen los resultados. Finalmente, se discuten los resultados y se proponen futuras líneas de investigación.

\title{
Presión fiscal
}

Los estados modernos se caracterizan por tener altos índices de presión fiscal, índices que han ido en alza en las últimas décadas (Berry \& Berry, 1994). En este escenario, cabe preguntar si un incremento en los impuestos podría desatar un aumento de la evasión fiscal, o una baja de impuestos una disminución en la evasión. Según el modelo propuesto por Allingham y Sandmo (1972), el nivel de presión o impuesto es uno de los factores que determinan el cumplimiento fiscal, estableciendo que a mayor nivel de impuestos menores serán las declaraciones de los ciudadanos. En esta línea, algunos estudios han encontrado una relación positiva entre nivel de impuesto 
y el cumplimiento fiscal (p.e., Alm, Sánchez, \& De Juan, 1995; Feinstein, 1991). No obstante, otros estudios han evidenciado que el incremento de la evasión ante el incremento de los impuestos no es significativa (p.e., Baldry, 1987; Porcano, 1988). Estos resultados contradictorios evidencian la necesidad de nuevas investigaciones que estudien la interacción entre estas dos variables.

Para explicar la relación positiva entre presión y evasión fiscal, el modelo de Allingham y Sandmo (1972) sostiene las personas evaden más impuestos cuando incrementa la presión fiscal porque el beneficio económico es mayor al evadir en contextos de alta presión impositiva, en comparación con el beneficio de evadir cuando la presión impositiva es baja (maximización de la utilidad esperada). Mientras que otros investigadores ofrecen explicaciones alternativas. Por una parte, Hammar Jagers y Nordblom (2009) sostienen que las personas que piensan que los impuestos al ingreso son demasiado altos creen que la evasión es generalizada entre los ciudadanos y, por esto, tienden a evadir. Por otra parte, Duch y Solaz (2015) han encontrado un incremento de evasión cuando la presión fiscal aumenta del $20 \%$ al $30 \%$, y sostienen que a partir de este último valor la tasa de impuesto se percibe como inapropiada e inaceptable por parte de los contribuyentes.

En función de lo expuesto, en este estudio evaluamos el efecto de diferentes niveles de presión fiscal sobre el comportamiento de declaración de impuestos (Estudio 1). Hipotetizamos que altos niveles impositivos generarán menor declaración de ganancias en comparación a bajos niveles impositivos (Hipótesis 1).

\section{Diferencias transculturales}

El comportamiento fiscal es afectado por el contexto socio-histórico, económico y cultural. Específicamente, Schneider y Enste (2013) señalan que la declaración de las ganancias varía entre los países. Como ejemplo, Zhang, Andrighetto, Ottone, Ponzano y Steinmo (2016) observaron que los italianos evaden menos impuestos que los británicos cuando se mantienen constantes las instituciones fiscales, las normas de redistribución y las probabilidades de auditoría. Sin embargo, otros autores no han evidenciado diferencias transculturales. Esto es, Andrighetto, Zhang, Ottone, Ponzano, D'Attoma y Steinmo (2016) encontraron que no hubo diferencias estadísticamente significativas en el promedio de la tasa de evasión entre suecos e italianos.

Es importante señalar que Argentina y España se caracterizan por particulares sistemas tributarios. En Argentina, las personas físicas están sujetas al impuesto a las ganancias en tasas progresivas sobre su ingreso anual, con tasas que varían entre el 9\% y el 35\% (Ministerio de Hacienda, 2018). En tanto que, en España, las tasas de este impuesto varían entre el 24\% y el 43\% (Agencia Estatal de Administración Tributaria, 2017). En ambos casos, mientras que para los trabajadores con un sueldo fijo (asalariados) la deducción de este porcentaje se realiza de manera 
automática, para aquellos individuos que no poseen un ingreso fijo mensual (autónomos) la declaración recae en el individuo, quien debe realizar la declaración de ganancias $\mathrm{y}$, en consecuencia, tienen más posibilidades de evadir.

En Argentina, la evasión del impuesto a las ganancias alcanzó el 49.7\% en 2010 según datos del Ministerio de Hacienda, y en el mes de mayo de 2016 significó un ingreso de 41695.5 millones de pesos de la recaudación tributaria (Ministerio de Economía y Finanzas Públicas). Mientras que España se ubica entre los 10 países con mayor evasión fiscal del mundo (Tax Justice Network, 2011). Además, los ciudadanos españoles perciben a la evasión fiscal como una conducta generalizada (Giachi, 2014). Si bien en ambos países se han realizado investigaciones que indagan el rol de distintas variables en el comportamiento fiscal de sus contribuyentes (ver p.e., Fortin, Lacroix, \& Villeval, 2007; Giarrizzo \& Sivori, 2010), según nuestro conocimiento, no existe un abordaje en términos comparativos entre ambos países.

Teniendo en cuenta la diferencia en los trasfondos culturales de cada país, en este trabajo comparamos la declaración de impuestos realizada por ciudadanos de Argentina y España (Estudio 1 y Estudio 2). En este sentido, esperamos observar diferencias en el comportamiento de declaración de impuestos entre argentinos y españoles (Hipótesis 2).

\section{Fuente del ingreso}

El estudio del comportamiento fiscal ha integrado progresivamente variables que no son exclusivamente económicas (Hammar et al., 2009; Kirchler, Hoelzl, \& Wahl, 2008). Mientras que la teoría económica clásica sostiene que los costos previos no deberían afectar las decisiones presentes, evidencia empírica demuestra que inversiones previas de dinero, tiempo y esfuerzo son relevantes para las decisiones presentes (Loewenstein \& Issacharoff, 1994). Estudios recientes han advertido que la forma en que las personas gastan su dinero puede estar relacionada con la forma en la que lo han obtenido (Carlsson, He, \& Martinsson, 2013; Cherry, Frykblom, \& Shogren, 2002). Ante esto surge el interrogatorio de si el origen o el esfuerzo invertido para obtener ganancias monetarias impactan en la posterior declaración de ganancias. Asumiendo la misma ganancia, igualdad en la presión impositiva y semejantes exigencias declarativas, ¿podríamos afirmar que una persona estaría igualmente dispuesta a declarar su ganancia si la obtuvo a través de un billete de lotería, la renta de un inmueble, la renta financiera o un trabajo remunerado? Si bien no son determinantes, existen resultados de estudios empíricos que aportan evidencia para responder esta pregunta.

Boylan y Sprinkle (2001) observaron que los participantes que ganaron su ingreso luego de resolver una tarea, presentaron mayor cumplimiento fiscal que aquellos que recibieron el ingreso directamente del experimentador. Esta diferencia se 
evidenció cuando el impuesto fue del $40 \%$, pero no hubo diferencias cuando fue del $20 \%$. Sagmeister et al. (2009) evaluaron las decisiones que tomaron los contribuyentes sobre el cumplimiento o evasión fiscal en distintos escenarios hipotéticos de esfuerzo de trabajo y encontraron que las personas que ganan su ingreso con poco esfuerzo son más propensas a evadir impuestos que cuando obtienen el ingreso a partir de mucho esfuerzo. Kirchler, Muehlbacher, Hoelzl y Webley (2009) también reportaron que la evasión fiscal es mayor en condiciones experimentales de bajo esfuerzo laboral.

A raíz de estos antecedentes comparamos el comportamiento impositivo entre ganancias obtenidas por desempeño (en una tarea de esfuerzo) y ganancias proporcionadas por el investigador (Estudio 1 y 2). Hipotetizamos una relación positiva entre esfuerzo y declaración de impuestos, con mayores niveles de cumplimiento fiscal cuando la ganancia es obtenida luego de realizar una tarea que implica esfuerzo en comparación a cuando es proporcionada por el investigador (Hipótesis 3).

\section{OBJETIVOS}

En síntesis, en este trabajo nos propusimos, por una parte, comparar la declaración de impuestos realizada por estudiantes universitarios de Argentina y España y, por otra parte, comparar el comportamiento impositivo entre ganancias obtenidas por desempeño (en una tarea de esfuerzo) y ganancias proporcionadas por el investigador en estudiantes universitarios de Argentina.

\section{MÉTODO}

En el marco de una investigación mayor, se desarrollaron los estudios comprendidos en este artículo, que implicaron experimentos. En el Estudio 1 participaron estudiantes universitarios de Argentina y España, quienes realizaron una tarea que permitió generar dinero de manera endógena. En cambio, el Estudio 2 sólo involucró participantes de Argentina y el dinero fue brindado por el experimentador. En ambos estudios los participantes indicaron cuánto declararían ante distintos niveles de presión tributaria. Para ello se utilizó el método de estrategia, en el cual los participantes tomaron decisiones condicionales para cada posible escenario de presión tributaria.

\section{Participantes}

En el Estudio 1, la muestra estuvo compuesta por estudiantes universitarios de 18 a 30 años de Psicología de la Universidad Nacional de Córdoba, Argentina, y de la Universidad Autónoma de Madrid, España. En Argentina participaron 105 estudiantes, de los cuales 100 completaron la información necesaria para ser incluidos en los análisis posteriores ( $27 \%$ varones, $72 \%$ mujeres; $M=20.42$ años, $D E=2.45$, 
1 persona no brindó información). En España participaron 114 estudiantes y 113 completaron toda la información necesaria $(28.3 \%$ varones, $71.7 \%$ mujeres; $M=$ 21.65 años, $D E=2.39)$. En el Estudio 2, por cuestiones de factibilidad, sólo participaron estudiantes de Argentina, específicamente 83 estudiantes de Psicología de la Universidad Nacional de Córdoba con edades entre 18 y 30 años. De esos estudiantes, 76 respondieron información completa y fueron incluidos en los análisis $(15.8 \%$ varones, $82.9 \%$ mujeres, 1 persona no brindó información; $M=21.04$ años, $D E=2.75)$.

\section{Tareas experimentales}

En este trabajo nos centramos en el nivel de declaración ante distintos niveles de presión tributaria, considerándose el monto ganado por cada participante (Estudio 1) u otorgado por el experimentador (Estudio 2). Específicamente, en el Estudio 1 los participantes resolvieron anagramas (Schweitzer, Odóñez, \& Douma, 2004), tarea utilizada para generar las Unidades de Dinero Experimental (UDE). La tarea consistió en la presentación de letras mezcladas que tenían que ser reorganizadas para formar palabras. Los participantes dispusieron de 5 minutos para resolver 5 anagramas de dificultad moderada (Jiménez \& Moreno, 2001); por cada anagrama resuelto obtenían 200 UDE. Al finalizar la tarea, tenían que indicar cuántos anagramas resolvieron y cuántas UDE ganaron. En tanto que en el Estudio 2 las UDE fueron provistas por el investigador, sin mediar tarea alguna por parte del participante.

En ambos estudios se empleó el método de la estrategia (Rauhut \& Winter, 2010) para recabar información sobre las decisiones de declaración ante distintos niveles de presión tributaria, a través de un cuestionario. Este método permite obtener información completa sobre las decisiones de los participantes ante diversas situaciones, lo que favorece la comprensión de la motivación conductual y la construcción de modelos descriptivos de la conducta (Mitzkewitz \& Nagel, 1993). A su vez, el hecho de pensar en reacciones posibles ante distintas situaciones facilita la comprensión del comportamiento en situaciones de la vida real, fuera del laboratorio, aportando así validez ecológica. A los fines de esta investigación, se evaluó la disposición a declarar ganancias ante los siguientes niveles de presión tributaria: $10 \%, 15 \%, 25 \%, 30 \%, 40 \%, 50 \%$. Las variables dependientes fueron las declaraciones de ganancia ante esos niveles de presión tributaria. Dado que en el Estudio 1 los participantes podían obtener como máximo 1000 UDE y en el Estudio 2 los participantes recibieron $10000 \mathrm{UDE}$, para considerar el monto que disponía cada participante al momento de la declaración y facilitar la comparación entre estudios, se consideraron como variables dependientes los puntajes relativos definidos por la cantidad de UDE declaradas sobre la cantidad de UDE iniciales (ganadas o recibidas). 


\section{Procedimiento}

En los Estudios 1 y 2 los participantes fueron convocados por invitaciones virtuales y presenciales en las facultades de Psicología de ambas Universidades. Las sesiones experimentales se realizaron en dichas facultades, con previa autorización de las autoridades correspondientes.

Durante la sesión experimental los participantes recibieron instrucciones y hojas de respuesta sobre: la tarea de generación de dinero (sólo estudio 1), el comportamiento de declaración ante distintos niveles de presión tributaria y datos socio-demográficos. Cada sesión fue conducida con un mínimo de diez participantes. En ambos países el experimentador a cargo fue de género masculino. Al finalizar la sesión experimental los participantes recibieron información sobre el experimento, se realizó el sorteo y recibieron el pago. Las decisiones de los participantes fueron incentivadas en ambos estudios. En cada sesión se sorteó 1 participante de cada 10 presentes en la sala; los participantes sorteados recibieron un monto de dinero en efectivo equivalente a las ganancias obtenidas en el experimento. Los participantes ganaron en promedio AR $\$ 168.85$ (Argentina - Estudio 1), €9.4 (España - Estudio 1) y AR \$57.71 (Argentina - Estudio 2).

\section{Análisis de datos}

Se realizaron pruebas de comparación de grupo paramétricas (MANOVA y ANOVA). A su vez, cuando se incumplieron alguno de los supuestos de esas pruebas se implementaron pruebas no paramétricas (test de Friedman, test de rangos con signos de Wilcoxon y test U de Mann-Whitney). Cuando se realizaron comparaciones múltiples no paramétricas se corrigió el $p$-valor según la cantidad de comparaciones realizadas. El nivel de significación fue .05 y se tuvo en cuenta el tamaño del efecto. La base de datos está disponible en https://osf.io/hbxj8/?view_only=d63fe8 b5da0b4894af99f63e42ede2d1.

Aspectos éticos

Los dos estudios se condujeron respetando los lineamientos éticos recomendados por la Asociación Psicológica Americana (American Psychological Association, 2010) y las normas bioéticas nacionales e internacionales (p.e., Declaración de Helsinki) sobre información de las características del estudio, confidencialidad y consentimiento informado.

\section{RESULTADOS}

\section{Estudio 1}

Primero se comparó el efecto de distintos niveles de presión tributaria sobre la declaración impositiva entre Argentina y España. El test de Mauchly indicó que no 
se cumplía el supuesto de esfericidad $\left(\chi_{14}^{2}=682.272, p<.000\right)$, por lo que los grados de libertad se corrigieron usando el estimador Greenhouse-Geisser $(\varepsilon=.37)$. El análisis evidenció un efecto principal del nivel de impuestos $(F(1.87,393.637)=$ $\left.132.44, p<.000, n^{2}=.386\right)$ y de interacción entre el nivel de impuestos y el país $\left(F(1.87,393.637)=23.657, p<.000, n^{2}=.101\right)$. No obstante, no hubo efecto principal de país $\left(F(1,211)=3.22, p=.074, n_{\mathrm{p}}^{2}=.015\right)$.

Luego se condujeron comparaciones por pares (con ajuste de Bonferroni), que evidenciaron que la declaración de ganancias disminuía significativamente a medida que se incrementaba la presión tributaria en ambos países. Al comparar los niveles de declaración de cada país por separado, en el caso de Argentina se observaron diferencias significativas en la declaración ante los distintos niveles de presión, excepto entre $10 \%-15 \%, 25 \%-30 \%$ y $40 \%-50 \%$. Mientras que en el caso de España se observaron diferencias significativas en la declaración entre todos los niveles de presión tributaria. A su vez, se observaron diferencias significativas entre los países cuando la presión tributaria era del $40 \%(p=.001)$ y del 50\% $(p<.000)$, mostrando España menores niveles de declaración en ambos casos (Tabla 1 y Figura 1). Vale señalar que debido al incumplimiento del supuesto de normalidad, se realizaron análisis no paramétricos, obteniéndose semejantes resultados.

\section{Tabla 1}

Declaración de ganancias según los niveles de presión fiscal y país

\begin{tabular}{|c|c|c|c|c|c|c|}
\hline $\operatorname{Tax} \%$ & Country & $\mathbf{N}$ & M & DS & Mdn & Rank \\
\hline \multirow{3}{*}{$10 \%$} & Argentina-Estudio 1 & 101 & 0.86 & 0.25 & 1 & 0.9 \\
\hline & Argentina-Estudio 2 & 76 & 0.85 & 0.23 & 1 & 0.95 \\
\hline & España & 114 & 0.91 & 0.21 & 1 & 2 \\
\hline \multirow{3}{*}{$15 \%$} & Argentina-Estudio 1 & 101 & 0.85 & 0.25 & 1 & 1 \\
\hline & Argentina-Estudio 2 & 76 & 0.81 & 0.24 & 0.9 & 0.96 \\
\hline & España & 114 & 0.87 & 0.25 & 1 & 2 \\
\hline \multirow{3}{*}{$25 \%$} & Argentina-Estudio 1 & 101 & 0.78 & 0.28 & 0.96 & 1.33 \\
\hline & Argentina-Estudio 2 & 76 & 0.7 & 0.27 & 0.76 & 0.99 \\
\hline & España & 114 & 0.79 & 0.27 & 0.89 & 1.75 \\
\hline \multirow{3}{*}{$30 \%$} & Argentina-Estudio 1 & 101 & 0.75 & 0.3 & 0.81 & 1.5 \\
\hline & Argentina-Estudio 2 & 76 & 0.66 & 0.27 & 0.7 & 0.99 \\
\hline & España & 114 & 0.69 & 0.28 & 0.75 & 1.5 \\
\hline \multirow{3}{*}{$40 \%$} & Argentina - Estudio 1 & 101 & 0.71 & 0.33 & 0.75 & 1.5 \\
\hline & Argentina-Estudio 2 & 76 & 0.59 & 0.28 & 0.6 & 0.98 \\
\hline & España & 114 & 0.57 & 0.29 & 0.53 & 1.25 \\
\hline \multirow{3}{*}{$50 \%$} & Argentina-Estudio 1 & 101 & 0.69 & 0.37 & 0.8 & 1.67 \\
\hline & Argentina-Estudio 2 & 76 & 0.56 & 0.29 & 0.5 & 0.99 \\
\hline & España & 114 & 0.48 & 0.3 & 0.5 & 1 \\
\hline
\end{tabular}

Nota. $\mathrm{N}=$ Número de casos; $\mathrm{M}=$ Media; $\mathrm{DS}=$ Desviación estándar; $\mathrm{Mdn}=$ Mediana 


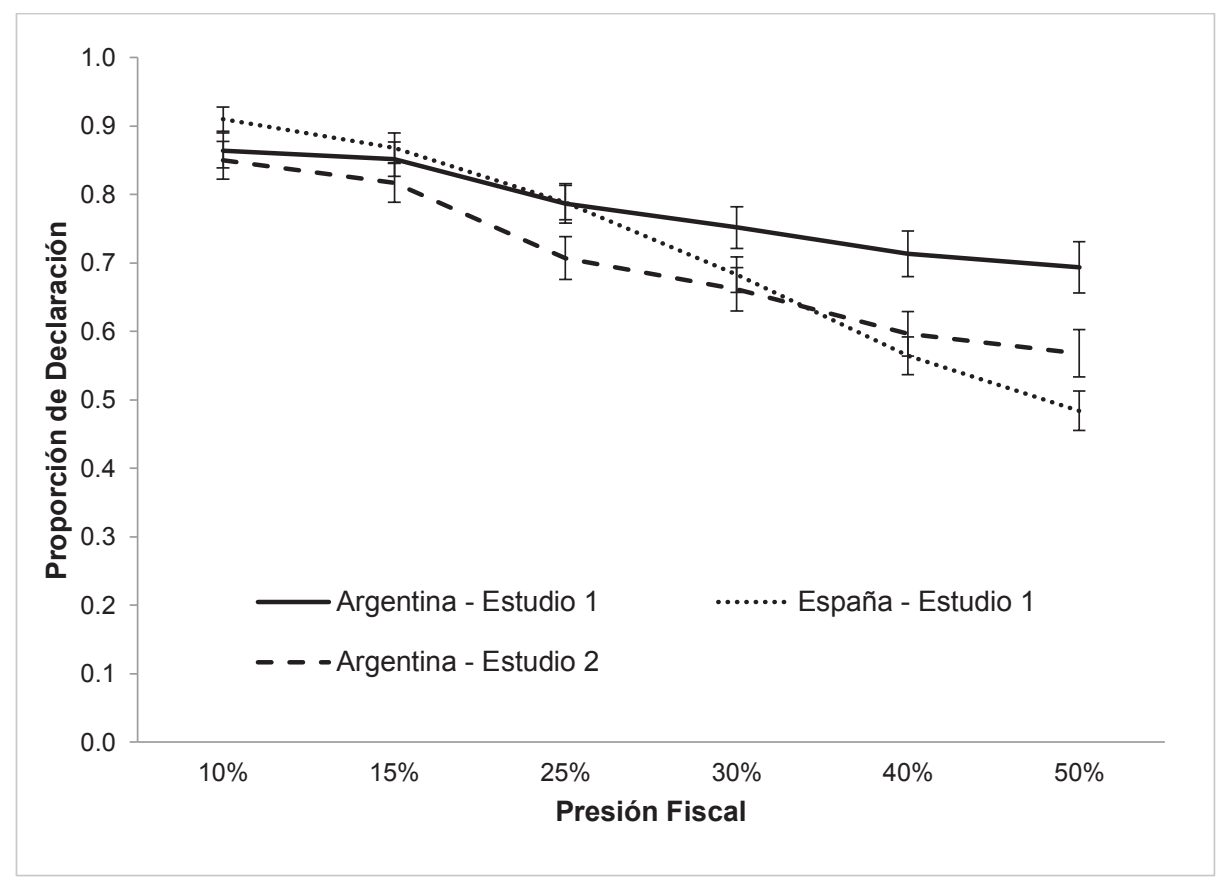

Figura 1. Declaración de ganancias (proporción) según los niveles de presión fiscal y país. Las barras muestran el error estándar de la media.

\section{Estudio 2}

En el segundo estudio sólo se recurrió a una muestra de estudiantes de Argentina. El test de Mauchly indicó que no se cumplía el supuesto de esfericidad $\left(\chi_{14}^{2}=\right.$ $167.477, p<.000$ ), por lo que los grados de libertad se corrigieron usando el estimador Greenhouse-Geisser $(\varepsilon=.47)$. El análisis evidenció un efecto principal del nivel de impuestos $\left(F(2.360,176.979)=45.877, p<.000, n_{p}^{2}=.380\right)$. Comparaciones por pares evidenciaron que a medida que se incrementó la presión tributaria, disminuyó la declaración de ganancias. Se observaron diferencias significativas entre todos los niveles de presión fiscal, excepto entre $10 \%-15 \%, 25 \%-30 \%$ y 40\%$50 \%$ (Tabla 1, Figura 1). Debido a que no se cumplió el supuesto de normalidad para algunas variables dependientes, se reiteraron los análisis utilizando técnicas no paramétricas. Se obtuvieron similares resultados y, además, resultó significativa la diferencia entre el porcentaje declarado entre $10 \%$ y $15 \%(Z=-3.323$, p < .001 , p-corregido $<.013$ ).

\section{Comparación Estudio 1 y 2}

Con el propósito de responder al objetivo sobre el rol de la fuente de dinero sobre el comportamiento fiscal, se compararon los resultados del estudio 1 y 2 (sólo muestras de Argentina). Vale recordar que en el Estudio 1 el monto a declarar 
había sido ganado tras la realización de una tarea experimental, mientras que en el Estudio 2 los participantes recibieron dinero del experimentador.

El test de Mauchly indicó que no se cumplía el supuesto de esfericidad $\left(\chi_{14}^{2}\right.$ $=475.940, p<.000$ ), por lo que los grados de libertad se corrigieron usando el estimador Greenhouse-Geisser $(\varepsilon=.41)$. El análisis evidenció un efecto principal del nivel de impuestos $\left(F(2.034,353.966)=58.891, p<.000, n^{2}=.253\right)$ y de interacción entre el nivel de impuestos y el estudio $(F(2.034,353.966)=3.407, p<.033$, $\left.n_{\mathrm{p}}^{2}=.019\right)$. A su vez, se apreció un efecto principal de estudio $(F(1,174)=4.042, p$ $\left.<.046, n_{\mathrm{p}}^{2}=.023\right)$.

Comparaciones por pares (con ajuste de Bonferroni) mostraron que la declaración de ganancias disminuía significativamente a medida que se incrementaba la presión tributaria, sin diferencias significativas entre los niveles de 10\%-15\% y 40\%-50\%. Las comparaciones para cada país fueron reportadas en los apartados previos. En tanto que al comparar los resultados de ambos estudios se observaron diferencias significativas a partir del $30 \%$ de presión tributaria. Concretamente, el nivel de declaración fue significativamente mayor en el Estudio 1 que en el Estudio 2 en los niveles de 30\% ( $p=.045), 40 \%(p=.014)$ y 50\% $(p=.018)$ (Tabla 1 y Figura 1). Debido al incumplimiento del supuesto de normalidad. En este caso, sólo resultó significativa la diferencia en el nivel del $40 \%$ de presión tributaria (U $=2917, \mathrm{p}<.008, \mathrm{p}$-corregido $<.045$ ).

\section{DISCUSIÓN}

El propósito de esta investigación fue analizar y comparar el rol de distintos niveles de presión tributaria sobre el comportamiento fiscal en estudiantes universitarios de Argentina y España. Para ello se condujeron dos estudios experimentales. En el primero, de carácter transcultural, se evaluó el efecto de diferentes niveles de presión fiscal sobre el comportamiento de declaración de impuestos, luego de realizar una tarea de obtención de dinero experimental. En el segundo estudio se comparó el comportamiento impositivo de participantes de Argentina cuando la ganancia a declarar era producto del desempeño en una tarea de esfuerzo (resultados del Estudio 1) y cuando era proporcionada por el investigador, nuevamente considerando distintos niveles de presión fiscal. En el Estudio 1 y en el 2 la declaración de ganancias disminuyó a medida que incrementaba la presión tributaria. Los resultados mostraron diferencias significativas en las declaraciones de los participantes de Argentina y España en altos índices de presión fiscal (40\% y $50 \%$ ). Además, se encontró que cuando el dinero es ganado mediante una tarea con esfuerzo, los participantes declaran significativamente más en comparación a cuando el dinero es brindado por el experimentador.

En el Estudio 1 observamos que la magnitud del impacto de diferentes niveles de presión fiscal varía entre Argentina y España. Es decir, se aprecian diferencias 
entre los países en la declaración ante distintos niveles de presión fiscal. Concretamente, se observó que la mayoría de las declaraciones se diferenciaron cuando se tuvo en cuenta el factor país, diferencias que fueron más evidentes en altos niveles de presión tributaria. A partir del $30 \%$ de presión tributaria las contribuciones de los participantes de España disminuyen abruptamente; las contribuciones de los participantes de Argentina también disminuyen, pero no con la misma magnitud. Cuando el nivel de impuesto alcanza el 50\% de las ganancias, las declaraciones de los participantes de España se reducen a la mitad de lo ganado, mientras que para los participantes de Argentina lo declarado nunca es menor a dos tercios de lo ganado.

En líneas generales los resultados están en consonancia con lo propuesto por Allingham y Sandmo (1972) y lo evidenciado en estudios previos (p.e., Alm et al., 1995; Feinstein, 1991) sobre la suba de la evasión ante el incremento de los impuestos. Los resultados muestran un mayor grado de tolerancia ante altos niveles de presión fiscal en Argentina. Mientras que en España parece haber un mayor grado de sensibilidad al cambio de presión fiscal, probablemente, debido a cierta estabilidad en las políticas fiscales del país. Futuros estudios podrían avanzar en esta línea y generar evidencia.

Se destaca la importancia de la variable presión fiscal a la hora de aplicar incrementos o disminución en los impuestos (política fiscal) a los fines de inducir el cumplimiento fiscal, ya que se espera que cuanto menor sea la presión fiscal mayor será el sinceramiento del comportamiento fiscal. A la vez, sería interesante considerar los efectos del tiempo y la gradualidad de los distintos cambios en los niveles de presión tributaria, aspectos no valorados en esta investigación.

Además, en el Estudio 1 se implementó una estrategia de generación de dinero endógena, esto es, los participantes tuvieron que completar una tarea a través de la cual ganaban dinero. Así, se pretendió emular el trabajo remunerado y dotar al estudio de mayor validez externa. Según Thaler (1985) diferentes fuentes de ingreso pueden generar diferentes modos de gastarlo. Particularmente, ganar dinero podría incrementar el costo percibido de reciprocar y por lo tanto disminuir su frecuencia (Anderson \& Putterman, 2006; Carpenter, 2007). En esta línea, Cherry et al. (2002) observaron que cuando el dinero era otorgado por el investigador las personas eran más cooperativas y apostaban menos a ganancias individuales, en comparación a cuando el dinero era ganado por el individuo. Según los autores, esto podría explicar la falta de comportamientos egoístas en la distribución de bienes públicos en el laboratorio. Carlsson et al. (2013) encontraron el mismo efecto. En resumen, dotar a los participantes de dinero experimental sin que medie un esfuerzo (o resolución de una tarea) podría alterar la validez externa de los resultados obtenidos en laboratorio y sobreestimar la frecuencia del comportamiento recíproco. 
En el Estudio 2, como en el Estudio 1, a medida que incrementó la presión tributaria disminuyó la declaración de ganancias. Al comparar la declaración ante distintos niveles de presión fiscal entre las muestras argentinas de los Estudios 1 y 2 se observó una tendencia semejante, aunque los niveles de cumplimiento en el Estudio 2 fueron más bajos, es decir, hubo mayor evasión. Las diferencias encontradas en la declaración podrían relacionarse con la forma de obtención de la dotación de dinero. En Estudio 1 el dinero se generó partir de una tarea de resolución de anagramas, mientras que en el Estudio 2 el participante recibió dinero sin realizar alguna tarea de esfuerzo. Estos resultados están en consonancia con lo observado en estudios previos (p.e., Boylan \& Sprinkle, 2001) Además, Sagmeister et al. (2009), en Argentina encontraron que las personas que ganan su ingreso con poco esfuerzo eran más propensas a evadir impuestos que cuando se esforzaban mucho. Los autores sostienen que, ante la probabilidad de detección y consecuente penalidad, los contribuyentes podrían ser reacios a perder su dinero ganado con esfuerzo, es decir que tenderían a mostrar mayor aversión al riesgo. Sin embargo, cabe señalar que la comparación con el trabajo de Sagmeister et al. (2009) no puede hacerse de forma directa debido a que en ninguno de los dos estudios implementamos castigos por evasión de impuestos. Por lo que futuras investigaciones podrían indagar la interacción entre la presión fiscal y el riesgo, generando un experimento que aplique detección y multas.

Antes de finalizar, cabe señalar algunas limitaciones del presente trabajo. Primero, en ambos estudios la muestra estuvo conformada por estudiantes universitarios. Al respecto, Wahl, Kastlunger, y Kirchler (2010) señalan que la mayoría de los estudiantes no están familiarizados con la tarea de pagar impuestos o declarar ganancias. Sin embargo, Alm et al. (1995) han observado que las decisiones de pago de impuestos son semejantes en estudiantes y no estudiantes. Segundo, el uso del método de la estrategia para obtener medidas de un mismo participante ante distintos niveles de presión tributaria podría generar un efecto de arrastre en las decisiones. Si bien esta estrategia resulta adecuada como una primera aproximación, sería interesante evaluar los efectos de los distintos niveles de presión tributaria a través de un diseño inter-sujeto. Tercero, cabe mencionar que los participantes en el Estudio 1 y 2 recibieron dotaciones iniciales diferentes. Específicamente, en el Estudio 2 el monto a declarar fue 10 veces mayor que el monto que se podía ganar en el Estudio 1 mediante la resolución de la tarea de anagramas. Aunque se generó una puntuación que permitiese comparar ambos estudios, probablemente las dotaciones diferentes pudieron generar una menor recaudación en el Estudio 1 que en el Estudio 2. Por último, es importante señalar que la comparación del comportamiento impositivo luego de recibir el dinero por esfuerzo o a través del investigador se realizó empleando diferentes muestras y en distintos momentos temporales. Futuros estudios podrían analizar si los resultados observados en el presente trabajo se replican empleando 
un diseño experimental inter-sujeto comparando las declaraciones entre quienes obtienen las ganancias por desempeño y quienes las reciben del investigador.

En síntesis, esta investigación remarca la importancia de tener en cuenta la variable presión fiscal y los aspectos socioculturales al momento de realizar cambios en las políticas fiscales. Asimismo, aporta evidencia sobre aspectos metodológicos en cuanto a la utilización/dotación del dinero en contextos experimentales.

\section{CONCLUSIONES}

Este artículo reporta los resultados de dos estudios que tuvieron como objetivo analizar el impacto del contexto socioeconómico, la presión fiscal y la fuente de dinero en la declaración de impuestos. Ambos estudios reportan una menor declaración de ganancias a medida que aumentó la presión fiscal, observándose una mayor tolerancia a altos niveles de presión fiscal en Argentina y una mayor sensibilidad a cambios en la presión tributaria en España. Adicionalmente, los estudios evidenciaron que cuando el dinero era ganado mediante una tarea con esfuerzo, las personas declaraban significativamente más en comparación a cuando el dinero era brindado por el experimentador. La evasión fiscal es un problema recurrente en numerosos países, siendo actualmente prioritario en la agenda de políticas públicas y en el campo académico. Estos resultados resaltan la importancia de considerar aspectos socioculturales y advertir sobre los riesgos de importar estrategias y políticas tributarias pensados para otros países, a la vez que destacan los efectos de la presión tributaria y la fuente de dinero en la declaración de ganancias.

\section{Agradecimientos / Acknowledgments:}

Al equipo "KuskaRuway-Investigación en Psicología y Economía Comportamental" por la colaboración en la fase de recolección de datos.

\section{Fuentes de financiamiento / Funding:}

Esta investigación contó con fondos provenientes de un subsidio de la Agencia Nacional de Ciencia y Tecnología (código: PICT 2014-1853) otorgados a Cecilia Reyna. Además, la recolección de datos en España se realizó mientras Lucas Tanos contaba con una beca del programa UAM-Santander.

\section{Rol de los autores / Authors Roles:}

LT: Conceptualización, Curación de datos, Análisis de datos, Metodología, Recolección de datos, Escritura (borrador original).

BS: Conceptualización, Curación de datos, Análisis iniciales, Metodología, Recolección de datos, Escritura (borrador original).

DM: Conceptualización, Metodología, Escritura (revision y edición). 
CR: Conceptualización, Búsqueda de financiamiento, Análisis de datos, Metodología, Supervisión, Escritura (borrador original), Escritura (revisión y edición).

\section{Aspectos éticos / legales; Ethics / legals:}

Los dos estudios se condujeron respetando los lineamientos éticos recomendados por la Asociación Psicológica Americana (American Psychological Association, 2010) y las normas bioéticas nacionales e internacionales (p.e., Declaración de Helsinki) sobre información de las características del estudio, confidencialidad y consentimiento informado.

\section{Conflicto de intereses / Competing interests:}

Los autores declaran que no existen conflictos de intereses.

\section{REFERENCIAS}

Agencia Estatal de Administración Tributaria (2017). Manual práctico, Renta 2017. https://www.agenciatributaria.es/static_files/AEAT/DIT/Contenidos_Publicos/CAT/ AYUWEB/Biblioteca_Virtual/Manuales_practicos/Renta/ManualRentaPatrimonio2017_es_es.pdf

Allingham, M., \& Sandmo, A. (1972). Income tax evasion: A theoretical analysis. Journal of Public Economics, 1, 323-338. https://doi.org/10.1016/0047-2727(72)90010-2

Alm, J., Sanchez, I. \& de Juan, A. (1995) Economic and noneconomic factors in tax complianc. Kyklos, 48(1), 3-18. https://doi.org/10.1111/j.1467-6435.1995.tb02312.x

American Psychological Association (2010). Ethical principles of psychologists and code of conduct.

Anderson, C. \& Putterman, L. (2006). Do non-strategic sanctions obey the law of demand? The demand for punishment in the voluntary contribution mechanism. Games and Economic Behavior, 54, 1-24. https://doi.org/10.1016/j.geb.2004.08.007

Andrighetto, G., Zhang, N., Ottone, S., Ponzano, F., D'Attoma, J., \& Steinmo, S. (2016). Evidence from a Tax Compliance Experiment in Sweden and Italy. Frontiers in Psychology, 7(472), 1-8. https://doi.org/10.3389/fpsyg.2016.00472

Baldry, J. C. (1987). Income tax evasion and the tax schedule: Some experimental results. Public Finance $=$ Finances publiques, 42(3), 357-383.

Berry, F. S., \& Berry, W. D. (1994). The politics of tax increases in the states. American Journal of Political Science, 38(3), 855-859. https://doi.org/10.2307/2111610

Bernasconi, M., Corazzini, L., \& Seri, R. (2014). Reference dependent preferences, hedonic adaptation and tax evasion: Does the tax burden matter? Journal of Economic Psychology, 40, 103-118. https://doi.org/10.1016/j.joep.2013.01.005 
Boylan, S. J., \& Sprinkle, G. B. (2001). Experimental evidence on the relation between tax rates and compliance: The effect of earned vs. endowed income. Journal of the American Taxation Association, 23(1), 75-90. https://doi.org/10.2308/jata.2001.23.1.75

Carlsson, F., He, H., \& Martinsson, P. (2013). Easy come, easy go: The role of windfall money in lab and field experiments. Experimental Economics, 16(2), 190-207. https:// doi.org/10.1007/s10683-012-9326-8

Carpenter, J. P. (2007). The demand for punishment. Journal of Economic Behavior \& Organization, 62(4), 522-542. https://doi.org/10.1016/j.jebo.2005.05.004

Cherry, T. L., Frykblom, P., \& Shogren, J. F. (2002). Hardnose the dictator. The American Economic Review, 92(4), 1218-1221. https://doi.org/10.1257/00028280260344740

Duch, R., \& Solaz, H. (2015). Why we cheat: Experimental evidence on tax compliance. Working Paper. Centre for Experimental Social Sciences, Nuffield College, University of Oxford.

Feinstein, J. S. (1991). An Econometric Analysis of Income Tax Evasion and its Detection. RAND Journal of Economics, 22(1), 14-35. https://doi.org/10.2307/2601005

Fortin, B., Lacroix, G., \& Villeval, M. C. (2007). Tax evasion and social interactions. Journal of Public Economics, 91(11-12), 2089-2112. https://doi.org/10.1016/j.jpubeco.2007.03.005

Giachi, S. (2014). Social dimensions of tax evasion: Trust and tax morale in contemporary Spain. Revista Española de Investigaciones Sociológicas, 145(1), 73-98. https://doi. org/10.5477/cis/reis.145.73

Giarrizzo, V., \& Sivori, J. S. (2010). La inconsistencia de la moral tributaria. El caso de los moralistas evasores. Pecvnia, 10, 95-124. https://doi.org/10.18002/pec.v0i10.641

Hammar, H., Jagers, S. C., \& Nordblom, K. (2009). Perceived tax evasion and the importance of trust. The Journal of Socio-Economics, 38(2), 238-245. https://doi. org/10.1016/j.socec.2008.07.003

Hofmann, E., Voracek, M., Bock, C., \& Kirchlerb, E. (2017). Tax compliance across sociodemographic categories: Meta-analyses of survey studies in 111 countries. Journal of Economic Psychology, 62, 63-71. https://doi.org/10.1016/j.joep.2017.06.005

Hyun, J. K. (2006). Tax Compliances in Korea and Japan: Why are they so different? Kore and the World Economy, 7(1), 135-153.

Jiménez, E. F., \& Moreno, J. B. (2001). Pesimismo defensivo, optimismo y dificultad de la tarea: El papel de las expectativas. Revista de psicología general y aplicada: Revista de la Federación Española de Asociaciones de Psicología, 54(3), 371-388.

Kirchler, E., Hoelzl, E., \& Wahl, I. (2008). Enforced versus voluntary tax compliance. The "Slippery Slope" framework. Journal of Economic Psychology, 29,210-225. https:// doi.org/10.1016/j.joep.2007.05.004

Kirchler, E., Muehlbacher, S., Hoelzl, E., \& Webley, P. (2009). Effort and aspirations in tax evasion: Experimental evidence. Applied Psychology, 58(3), 488-507. https://doi. org/10.1111/j.1464-0597.2009.00403.x 
Kogler, C., Batrancea, L., Nichita, A., Jozsef, P., Belianin, A., \& Kirchler, E. (2013). Trust and power as determinants of tax compliance: Testing the assumptions of the slippery slope framework in Austria, Hungary, Romania and Russia. Journal of Economic Psychology, 34, 169-180. https://doi.org/10.1016/j.joep.2012.09.010

Lewis, A., Carrera, S., Cullis, J., \& Jones, P. (2009). Individual, cognitive and cultural differences in tax compliance: UK and Italy compared. Journal of Economic Psychology, 30(3), 431-445. https://doi.org/10.1016/j.joep.2008.11.002

Loewenstein, G., \& Issacharoff, S. (1994). Source dependence in the valuation of objects. Journal of Behavioral Decision Making, 7(3), 157-168. https://doi.org/10.1002/ bdm.3960070302

Ministerio de Hacienda (2018). Tributos vigentes en la República Argentina. Recuperado de https://www.argentina.gob.ar/sites/default/files/tributos_vigentes_al_31-12-18.pdf

Mitzkewitz, M., \& Nagel, R. (1993). Experimental results on ultimatum games with incomplete information. International Journal of Game Theory, 22(2), 171-198. https:// doi.org//10.1007/BF01243649

Porcano, T. M. (1988). Correlates of tax evasion. Journal of economic psychology, 9(1), 47-67. https://doi.org/10.1016/0167-4870(88)90031-1

Rauhut, H., \& Winter, F. (2010). A sociological perspective on measuring social norms by means of strategy method experiments. Social Science Research, 39(6), 1181-1194. https://doi.org/10.1016/j.ssresearch.2010.06.009

Sagmeister, E., Muehlbacher, S., \& Kirchler, E. (2009). La influencia del nivel de exigencia y del esfuerzo puesto en el trabajo sobre la evasión de impuestos en Argentina. Perspectivas en Psicología, 6, 66-71.

Schneider, F., \& Enste, D. H. (2013). The shadow economy. An international survey. Cambridge: Cambridge University Press.

Schweitzer, M. E., Ordóñez, L., \& Douma, B. (2004). Goal setting as a motivator of unethical behavior. Academy of Management Journal, 47(3), 422-432. https://doi. org/10.2307/20159591

Slemrod, J., \& Yitzhaki, S. (2002). Tax avoidance, evasion, and administration. Handbook of Public Economics, 3, 1423-1470. https://doi.org/10.3386/w7473

Tax Justice Network (2011). The cost of tax abuse. Recuperado de http://www.tackletaxhavens.com/Cost_of_Tax_Abuse_TJN\%20Research_23rd_Nov_2011.pdf

Thaler, R. H. (1985). Mental accounting and consumer choice. Marketing Science, 4(3), 199-214. https://doi.org/10.1287/mksc.4.3.199

Wahl, I., Kastlunger, B., \& Kirchler, E. (2010). Trust in authorities and power to enforce tax compliance: An empirical analysis of the "Slippery Slope Framework". Law \& Policy, 32(4), 383-406. https://doi.org/10.1111/j.1467-9930.2010.00327.x

Zhang, N., Andrighetto, G., Ottone, S., Ponzano, F., \& Steinmo, S. (2016). "Willing to Pay?" Tax Compliance in Britain and Italy: An Experimental Analysis. PLOS ONE, 11(2), 1-14. https://doi.org/10.1371/journal.pone.0150277 